\title{
Acquired Prescription Drug Behaviors
}

\author{
Jerrold Rand* \\ Training Facility for internship for psychologist, USA
}

Submission: October 28, 2017; Published: November 15, 2017

*Corresponding author: Jerrold Rand, Training Facility for internship for phycologist, USA, Email: jerrynrand@gmail.com

\section{Abstract}

Functional restoration requires a team approach. Behaviors that occur in patients with chronic pain, disability and prescription drug use. Those results in problematic prescription drug use, during the ongoing process of medical care, are prone to develop behaviors that appear in the doctor patient relationship. Such behaviors can be addressed independently of conventional addiction treatment. Rehabilitation both from emotional and physical function requires an understanding of the chronic illness and prior medical care that may be the foundation of behaviors leading to treatment for Addiction and Rehabilitative Medicine. Patients can arrive years or decades after injuries or illnesses began. Ongoing prescribing of Opioids can form certain behaviors related to ongoing prescribing by Doctors and receiving medications from patients and affect the Doctor patient relationship. These behaviors that can be seen in Addiction Medicine and Rehabilitative medicine combined treatment.

\section{Introduction}

Treatment providers in hospitals and clinics are trained to provide a structured treatment derived from a pattern and rules developed by the organizational hierarchy. The resultant behavioral responses from the Doctor, patient interaction evolves as treatments progress. These contacts, actions and responses results in a system of patients acquiring help and of treatment providers prescribing habits in controlled substance medicallegal treatment encounter. The rules and regulations that have evolved in prescribing medications tent to follow a process of regulatory form, as opposed to functional improvement response. When regulatory treatment do not meet regulatory needs, there can be a disconnect in treatment outcome .Historically, physicians are trained to write a prescription in response to a complaint or diagnoses of a disease process, and patients have an expectation of acquiring a prescription from the encounter. Non-prescription compounds or medications that are in the arena of OTC, (over the counter medications/nutrients) may be omitted from traditional medicine, and then fall into complementary or alternative medicine formats.

What follows is meant to increase understanding, and decrease mistrust to the institutional format of treatment. Many behaviors remain unidentified or create mistrust by all parties involved. To some extent, the patterns and expectation of ongoing illness and traditional outcomes becomes part treatment and evolution of illness. This lack of insight may result in lack of intervention in the vicious cycle of disease. High-risk settings of addiction, pain, and psychiatric illness present as a high-risk encounter. Improved behavioral understanding can result in transparency of medical legal concerns and defensive medicine that is ever increasing in regulated controlled structured treatment. Many issues involve the use of controlled substances and their potential inherent properties. Clinicians need to be comfortable in the use of medications for illness and pain, and be aware of medication use patterns in clients with a history of addiction. In some cases, Opioids have not been prescribed because of unwanted concerns about addiction. We are concerned that behavior patterns, with the appearance of drug abuse or addiction, can be labeled as drug addiction, unfortunately ensnaring in the process, victims who are motivated to engage in these learned and acquired survival behaviors (due to the ravages of pain) in an attempt to cope with their lives.

When viewing these Acquired Prescription Drug Behaviors (APDB), the term "addiction" creates diagnostic confusion, resulting in a wide spectrum of diagnostic meaning. As a result, it can become necessary to consider the social context in which the diagnosis of addiction is made in pain patients. A typical government sponsored edict entitled "War on Drugs" generates an atmosphere of fear and panic to main line clinicians across America. Chronic Pain patients on opioid treatment are frequently seen as the causative factor of the Opioid epidemic. Clinicians and insurance adjusters are eager not to overlook any behavior that could later be pointed to as evidence that an Opioid addict may be missed. This can create the impression of addiction, and an altered approach to treating that can be detrimental to the pain suffer 


\section{a) Defining the Problem}

Clients with drug addiction and uncontrolled pain commonly have persistent intolerable pain, because of the multi-factorial complex issues of pain control and management. Physicians should be concerned about writing prescriptions to those whose sole purpose is to acquire controlled substances for personal addictive use or diversion. This caution of treating clients with illegal behaviors should not interfere with treating people with (APDB) It is also not uncommon that drug addicts with or without criminal behaviors have significant pain disorders. Acquired prescription drug behaviors can occur in individuals with and without prior genetic, or psycho-social issues, or in the previously and/or currently alcoholic and/or addicted individuals, or can evolve later during the course of treatment of these clients. The Challenge of Opioid treatment extends to Opioid abuse, diversion and overdose. It involves treatment for chronic pain and addressing the amount of the substances present and readily available in society.

\section{b) The Evolution of Acquired Prescription Drug Behaviors (APDB's)}

When pain is not in a tolerable range, it remains a trigger for aberrant drug related behaviors and potential drug problems or overdose. In this day of escalating Opioid overdoses and abuse, we look at pain treatment as a significant cause of the current Opioid epidem

\section{c) Chemical Coping}

Evolution of APDB's clinically appear most commonly in the same formative age group as addictive disorders (15 to 25 years of age), and are more apparent when illness occurs earlier in childhood and when more severe. Behaviors evolve when pain, discomfort, and/or psychological effects of ongoing illness persist resulting in learned behavior patterns. During initial or ongoing trauma or medical illnesses, "needs" are not fulfilled or are mainly met through therapeutic pharmacologic treatments; this series of events can cause developmental imprinting (more pronounced in formative developmental stages in early years). The development of emotional coping with an illness, when combined with medication management to treat discomforts by caring physicians, can be termed "chemical coping" These behaviors, once needed to cope and survive the trauma of illness persist, can become pathological and result in ongoing abnormal behaviors or "chemical coping."

These APDBs may begin as coping mechanisms to get the attention of family, friends, or physicians in order to get their perceived needs met. The very act of medicating physical or emotional feelings provides positive reinforcement for continued drug use for any physical and ultimately can be used to medicate emotional symptoms. As a result, behaviorally the younger a patient starts with pain and medication, the more likely that behavior becomes ingrained. The illness itself becomes the cause for a traumatic psychic injury. This occurs during formative periods of development and or correlate with ongoing illness and psychosocial loses, and can lead to a "behavioral homeostasis" as part of that phase of development.

Patients develop patterns of getting attention or acquire comfort medications for physical pain or discomfort, and emotional relief. These coping skills may be protective and allow for survival from the enormity of the impact of illness (pain, depression, sleep, fear, anxiety and panic). These coping skills are frequently necessary to get through the initial phases of illness and as such are "acquired" during the course of illness and influence this stage of emotional development. As time evolves, these behavior patterns may be modified by periods of exacerbations during medical treatments, disease progression or break through pain and if relief is perceived to be delayed or not coming, pseudo addiction behavioral responses occur. In addition, dealing with life's intolerable discomforts, the evolution of negative coping skills and negative self-talk become pattern behaviors. These individual behaviors evolve into a host of aberrant drug related behaviors, which may represent selfmedicating to get individual needs met. The patient feels that they are not adequately taken care of and respond by learning ways to get their medical needs met. These are viewed as aberrance of behaviors and may achieve the form of classic addiction. Now, the client may be very unaware of anything other than their attempts to cope. What happens next depends on the response of outside forces and how the future developments of behaviors are molded! The treating physician becomes part of this ongoing process. A greater understanding of the relationship and partnership of physician and patient may be needed to optimize care. Additional treatment can be aimed at identifying abnormal behaviors, and processing the origins of behavior in order to alter future interactions and empower individuals into a safer future. The patient gains the ability set his own limits of drug use with quality of life and personal safety being the priority.

There are programs that understand and work as a team on behavioral pain management, while stabilizing the biological and behavioral elements to achieve homeostasis. As practitioners, we are concerned about the issues of diversion and sales, which can occur even in pain patients. However, prescription abuse issues, occurring in patients being treated for pain, may be best treated as adverse effects of medications, which may present as behavior issues, and physiological impairments and tolerance, as treatable conditions. Discharge planning goals are to correct or re-establish new physician relations for future care upon transition.

\section{Self-medicating}

The corollary, we must realize that self-medicating occurs in both pain and addictive states. Self-medicating is common 
in the dual diagnosis population, as well as in the pain and dual diagnosis combined disorders. The behavioral approach and treatment of the individual's psychological issues can be addressed. Acceptance becomes the corner stone of treatment in the combined field of pain and addiction.

Behaviors result from processing incoming feelings, using developing defense mechanisms and patterns of denial. Prescribing physicians can become aware of aberrant behaviors that are occurring. Skills are needed to interpret these behaviors in order to continue to prescribe controlled medications. The challenge is to interpret and gauge the need for different medications, and what amounts are needed. These incoming messages assist the prescriber in making a judgment on the drugs and amounts given.

Treatment decisions can be difficult to see through a series of psychological events that make it difficult for us to interpret what the clients' needs are. In the end we are sometimes faced with relying on the distorted signals, amplifications, minimizations, partial truths, and at times only hear the patients' interpretation through these distortions. Impaired self-awareness may result in a state of denial were even the patient is unaware of the reality of the quality, quantity or locations of their own bodily discomforts. This later distortion of bodily awareness although rare is a not uncommon finding in the extreme co-occurring illness

\section{Drug Seeking Behaviors}

Pseudo addiction represents drug-seeking behaviors created by the patient's fear and perception that their pain relief is inadequate. The term "pseudo addiction" was coined in 1989, to describe chronic pain victims mistakenly diagnosed as suffering from opioid addiction after they were driven, by under-treated pain, to display certain drug seeking behaviors. The misconception of the term addiction can harm the patient suffering from pain. In reality, both drug addicts and non- addicts may have pseudo addiction. When patients display certain med seeking behaviors; they may unjustly be denied a necessary and appropriate change of medications or any further treatment at all. To complicate matters, similar behavioral patterns seen in addiction may evolve creating a behavioral pseudo addiction (BPA), even after the pain is correctly managed.

\section{Coping Skills}

To understand how aberrant drug related behaviors affect the patient, we need to look at their harsh realities of treatment, and the responsibility that many professionals share. Negative and positive reinforcement, in part, are nurtured by the medical and/or clinical helping professions. Over the length of a genuine physical malady that may last for years, the development of Acquired Prescription Drug Behaviors (APDB) evolves. These APDB behaviors are, at times, mistaken for addiction. With or without a trained eye, it is difficult to differentiate between the two behaviors, learned and/or addictive. In fact, these behaviors can be identical in presentation with classic addictive behaviors. Frequently, the patient has no knowledge of the (APDB), is unaware and, in effect, in a pattern of denial. What have evolved from positive well-meaning coping devices that may have been life saving has now become negative coping devices. This behavioral Homeostasis "homeostasis through adaptation" has now become a negative adaptation (negative coping skills) manifested by these acquired aberrant behaviors.

With treatment, obtaining new attitudes and behaviors that lead to more appropriate coping skills become the groundwork for recovering from the destructive consequences of pain. Accusing and punishing can make the process worse. Negative aberrant behaviors that continue to evolve can progress or cross over to addiction, particularly in the predisposed or sensitized individuals. The purpose of treatment is to restore the clients to a functional, satisfying existence, where the pain does not dominate their lives. In the process, drug abuse and addictive behaviors can be effectively addressed and dealt with in a way that leads to stabilization.

Functional restoration requires a team approach. Behaviors that occur in patients with chronic pain, disability and problematic drug use who are involved prescription drugs use, during medical care, are prone to develop behaviors that appear in the doctor patient relationship. Such behaviors can be addressed these behaviors independently of conventional addiction treatment. Rehabilitation both from emotional and physical function, requires an understanding of the chronic illness and prior medical care that may be the foundation of behaviors leading to treatment for Addiction and Rehabilitative Medicine. 
$\begin{array}{ll}\text { (C) } & \text { This work is licensed under Creative } \\ \text { Commons Attribution 4.0 License }\end{array}$
- Swift Peer Review

- E-prints Service

- Manuscript Podcast for convenient understanding

- Global attainment for your research

- Manuscript accessibility in different formats

( Pdf, E-pub, Full Text, Audio)

- Unceasing customer service

Track the below URL for one-step submission https://juniperpublishers.com/online-submission.php 